Distribution Category:

Magnetic Fusion Energy

(UC-420)

ANL/F PP/TM- -256

DE92 000491

ANL/FPP/TM-256

ARGONNE NATIONAL LABORATORY

9700 South Cass Avenue

Argonne, Illinois 60439-4801

\title{
INDUCTIVE CURRENTS IN AN RF DRIVEN PLASMA
}

Jon Kinsey and David A. Ehst

Fusion Power Program

August 1991

\footnotetext{
Work supported by

Office of Fusion Energy

U.S. Department of Energy

Under Contract W-31-109-Eng-38
} 


\section{TABLE OF CONTENTS}

\section{$\underline{\text { Page }}$}

Abstract

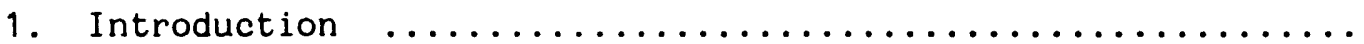

1

2. Self-Consistent Equilibria with Inductive Currenis $\ldots . \ldots \ldots$.

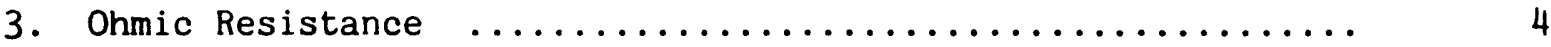

3.1 Resistance in an RF Plasma $\ldots \ldots \ldots \ldots \ldots \ldots \ldots \ldots \ldots \ldots \ldots \ldots \ldots \ldots .1$

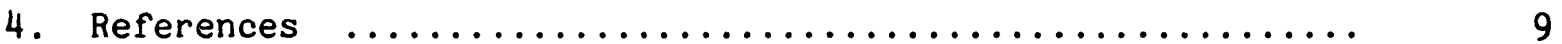




\section{LIST OF FIGURES}

$\underline{\text { Page }}$

Figure 1 RFCD/MHD equilibrium for DIII-D with $\mathrm{T}_{\text {eo }}=3.5 \mathrm{keV}$,

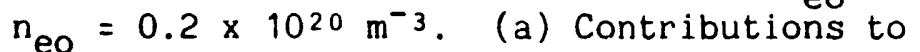
$\left\langle\mathrm{J}_{\text {B }}\right.$ > from FWCD (dashed), bootstrap (chain-dashed), inductive + boostrap (chain-dotted), and total (dotted). (b) Components of toroidal current density. (c) FW ray

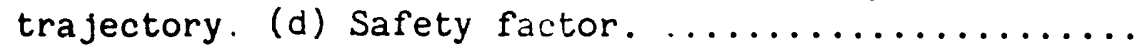

Figure 2 Coulomb Logarithm. $\ldots \ldots \ldots \ldots \ldots \ldots \ldots \ldots \ldots \ldots \ldots \ldots \ldots \ldots \ldots$

Figure 3 Comparison of analytic and numerical resistance. ....

\section{LIST OF TABLES}

$\underline{\text { Page }}$

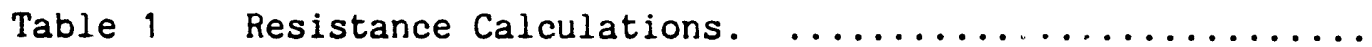

Table 2 Resistance Calculations. 


\title{
INDUCTIVE CURRENTS IN AN RF DRIVEN PLASMA
}

by

\author{
Jon Kinsey* and David A. Ehst
}

\begin{abstract}
Inductive effects are included in a self-consistent current drive model for axisymmetric tokamak plasmas used in the two-dimensional current drive/MHD equilibrium code, RIP. Previous simulations of current driven equilibria allowed for the steady-state calculation of bootstrap and RF currents. The addition of an inductive current is applied to enhance accurate design and interpretation of tokamak experiments. A convenient expression for the ohmic resistance in a tokamak plasma is derived to aid in the design of reactor grade MHD equilibria.
\end{abstract}

\section{INTRODUCTION}

According to Faraday's law, an electric field arises when there is a changing magnetic field. This is of particular interest in tokamak startup experiments in which the plasma current is gradually ramped up to its nearly steady-state values. This study calculates the inductive current driven by an electric field generated in a tokamak plasma from a changing flux through the torus. During the pulse length of the $\mathrm{OH}$ (primary) coils most, if not all, of the total plasma (secondary) current is supplied inductively until the maximum allowable magnetic flux is reached in the $\mathrm{OH}$ coils. Even after the appearance of bootstrap and an RF seed current, the inductive current can be significant as the plasma enters the steady-state regime.

* Permanent address Nuclear Engineering Department, University of Illinois. 


\section{SELF-CONSISTENT EQUILIBRIA WITH INDUCTIVE CURRENTS}

Previously, a theory for non-inductive current drive was proposed for steady-state tokamak operation $[1,2]$. This theory has now been extended to include the presence of a weak DC electric field, E. This assumes little or no runaway electron generation and that the effects of an emf induced current and RF driven current are additive. The total current density is written as $\mathrm{J}_{\mathrm{TOT}}=\mathrm{J}_{\mathrm{OH}}+\mathrm{J}_{\mathrm{RF}}$ where the ohmic current is the current induced without $\mathrm{RF}$ power present and $J_{R F}$ is the $R F$ current in the absence of an electric field. The addition of another term, $\sigma_{1} E$, has been described by Fisch[3] where the "hot" conductivity allows for a nonlinear dependence on both inductive and $\mathrm{RF}$ effects. Although comparatively small for the cases we consider, the hot conductivity term may warrant consideration in future studies. The electron distribution function $f$ may be solved in toroidal geometry using the steadystate equation

$$
v_{\|} \hat{B} \cdot \nabla f-C(f)=-\frac{e E_{\|}}{m} \frac{\partial f}{\partial v_{\|}}-\underline{v}_{d} \cdot \nabla f+\frac{\partial}{\partial \underline{v}} \cdot \underline{r},
$$

where the three terms on the r.h.s. are the electric field, gradient drift, and RF heating drivers, respectively. This determines the individual currents contributing to $\mathrm{J}_{\|}$(current density parallel to the magnetic field $\underline{B}$ ) such that

$$
\frac{\left\langle\mathrm{J}_{\|} \mathrm{B}\right\rangle}{\left\langle\mathrm{B}^{2}\right\rangle}=\mathrm{G}(\psi)+\mathrm{H}(\psi)+\mathrm{I}(\psi)
$$

where <> is a flux surface average[2], and $G(\psi), H(\psi)$, and $I(\psi)$ describe the $R F$, bootstrap, and inductive effects as a function of poloidal flux. The flux $\downarrow$ is the solution to the Grad-Shafranov equation, 


$$
R^{2} \nabla \quad\left[\frac{\nabla \psi(R, Z)}{R^{2}}\right]=-4 \pi^{2} \quad\left[\mu R^{2} \frac{d p}{d \psi}+F \frac{d F}{d \psi}\right],
$$

Here, $p(\psi)$ is the plasma pressure, $F(\psi)=\mathrm{RB}_{\mathrm{T}}$ is the plasma diamagnetism, $\mu$ is the permeability of free space, and $B_{T}$ is the toroidal magnetic field. $F(\psi)$ is found by solving

$$
\frac{d F}{d \psi}+\frac{\mu F}{\left\langle B^{2}\right\rangle} \frac{d p}{d \psi}=\frac{-\mu}{2 \pi}[G(\psi)+H(\psi)+I(\psi)]
$$

through successive iterations until satisfactory convergence is reached.

The three current density functions are computed in RIP as follows. The RF driven current, $G$, is calculated using the function in Ref. 4, which accounts for reductions in RFCD efficiency at arbitrary aspect ratio due to magnetic trapping. Ray tracing and wave damping are computed in RIP in order to find $G(\psi)$. The bootstrap contribution, $H$, is found by interpolating between the collisionless formulation[5], valid at arbitrary aspect ratio and for artitrary ion mixture, and the Hinton-Hazeltine expression[6] at high collisionality. The iwuuctive current is given by $I=\sigma_{\text {eff }}\left\langle E_{\|} B\right\rangle \mid\left\langle B^{2}\right\rangle$, and off on each flux surface is computed from either the Hirshman-Sigmar[5] or the Hinton-Hazeltine[6] expressions. Since reactor tokamaks are well into the banana regime the former evaluation of $\sigma_{\text {eff }}$ is used in this study, and this is valid at arbitrary aspect ratio. Also, assuming the internal inductance is not changing (which is generally not valid during transients) the electric field takes on a simple $1 / R$ dependence, $\left\langle E_{\|} B\right\rangle=\left\langle V_{L} / 2 \pi\right\rangle\left\langle B_{T} / R\right\rangle$, in our present treatment. We denote the loop voltage as $V_{L}$.

Figure 1 depicts a RIP equilibrium solution for a DIII-D plasma in the presence of an inductive c.urrent and a single FW ray at $60 \mathrm{MHz}$ launched from the outboard midplane. The parameters used are: $R_{0}=1.7 \mathrm{~m}, a=0.7 \mathrm{~m}, \kappa=$ 
$1.6, d=0.40, V_{L}=0.036 \mathrm{~V}, z_{\text {eff }}=1.5, I_{p}=262 \mathrm{kA} B_{T}=1.09 \mathrm{~T}$ and $P_{R F}=$ $300 \mathrm{~kW}$. At the plasma core, the inductive current is shown to have significant effect on the total current.

\section{OHMIC RESISTANCE}

For a circular concentric plasma, the DC resistance can be approximated from the equation

$$
\begin{aligned}
R & \simeq 2 \pi R_{0}\left[\int_{0}^{\psi b} d r_{\psi} \oint \sigma_{e f f} d r{ }_{x}\right]-1 \\
& \simeq 2 \pi R_{0}\left[\int_{0}^{2 \pi} \int_{0}^{a} \sigma_{e f f} r d r d \theta\right]-1
\end{aligned}
$$

The effective parallel conductivity is found using an expression by Wesson[7],

$$
\sigma_{\|}=6.06 \times 10^{8} \frac{\mathrm{T}^{3 / 2}}{\mathrm{~g}(2) \ln \Lambda}
$$

where $g(Z)$ is determined approximately in terms of the effective ion charge $Z$ and a resistivity factor $\mathrm{N}(\mathrm{Z})$ such that

$$
g(Z)=N(Z) Z:
$$

\begin{tabular}{lllll}
$z$ & 1 & 2 & 4 & 16 \\
\hline $\mathrm{N}$ & 1.0 & 0.85 & 0.74 & 0.63
\end{tabular}

The temperature profile is specified by the normalized poloidal flux raised to a power: 


$$
\mathrm{T}=\mathrm{T}_{0} \tilde{\psi}^{\alpha_{\mathrm{T}}}
$$

Here, $\tilde{\psi}=\left(\psi_{-}-\psi_{b}\right) /\left(\psi_{a}-\psi_{b}\right)$, with $\psi_{a}$ and $\psi_{b}$ as the poloidal fluxes at the magnetic axis and boundary, respectively. The profile width is determined by the peaking factor, $a_{\mathrm{T}}$.

Solving now for $R$, and taking $\psi / \psi_{b} \approx(r / a)^{2}$,

$$
R=\frac{g(z) \ln \Lambda\left(3 \alpha_{T}+2\right)}{6.06 \times 10^{8}} \frac{R_{0}}{a^{2}} T_{e o}^{-3 / 2}
$$

where $a$ is an effective minor radius. We evaluate the Coulomb logarithm as

$$
\ln \Lambda=15.2-\frac{1}{2} \ln \left(n_{e}\right)+\ln \left(T_{e}\right)
$$

where the electron density, $\mathrm{n}_{e}$, has units of $\left(10^{\left.20 / \mathrm{m}^{3}\right)}\right.$, and $\mathrm{T}_{e}$ is in $\operatorname{keV}[7]$. Figure 2 shows the Coulomb $\log$ for $n_{e}$ vallies of interest.

Taking $\alpha_{\mathrm{T}}=1.275$, for example,

$$
R=9.6 \times 10^{-9} \frac{g(z) R_{o} T_{e o}}{a^{2}} \text { ln } \Lambda .
$$

A comparison can then be made to values determined from the RIP code using Ohm's law,

$$
R=\frac{V_{L}}{I_{I N}}=\frac{V_{L}}{I_{P}-I_{B S}} ;
$$

note that the bootstrap contribution is subtracted from the total current (and no $R F$ current is driven). Here, $V_{L}$ is the loop voltage, $I_{p}$ is the plasma current, $I_{B S}$ is the bootstrap driven current, and $I_{I N}$ is the inductive 
current. Table 1 shows that while equation (11), an approximate analytic expression, produces comparable values to the numerical results in some places, it fails to account for variances in the plasma resistance associated with different temperature profiles.

We can account for the temperature profile effect to some extent. The volume averaged electron temperature is roughly

$$
\bar{T}_{e}=\frac{\int_{0}^{a} T_{e o}\left(1-r^{2 / a^{2}}\right)^{\alpha_{T}} r d r}{\int_{0}^{a} r d r},
$$

So,

$$
\frac{\overline{\mathrm{T}}_{\mathrm{e}}}{\mathrm{T}_{\mathrm{eo}}}=\frac{1}{1+\alpha_{\mathrm{T}}} \text {, }
$$

Therefore, correlating $a_{\mathrm{T}}$ to $\mathrm{T}_{e_{0}} / \overline{\mathrm{T}}_{\mathrm{e}}$ in Eqs. (9) and (14) the resistance is found to vary as

$$
R-\left(3 \frac{T_{e o}}{\bar{T}_{e}}-1\right) T_{e o}^{-3 / 2},
$$

For a single value of $\mathrm{T}_{e o}$, the resistance decreases as $\overline{\mathrm{T}}_{e}$ increases. This behavior appears to match the output of the RIP code.

Next, we wish to include the trapped particle effects into our calculation. Using the Hinton-Hazeltine fit for an arbitrary aspect ratio tokamak in the banana regime,

$$
\begin{aligned}
\sigma_{\text {eff }} & =\sigma_{\|}(1-1.95 \gamma \varepsilon+0.95 \varepsilon), \\
& \equiv f(\varepsilon) \sigma_{\|} .
\end{aligned}
$$


Somewhat arbitrarily we take $\varepsilon=1 / 3 \mathrm{~A}$, with $\mathrm{A}$ denoting the aspect ratio. The resistance now becomes

$$
R=\frac{9.6 \times 10^{-9} g(Z) R^{*} R_{0}}{f(\varepsilon) x} \ln \Lambda
$$

Here,

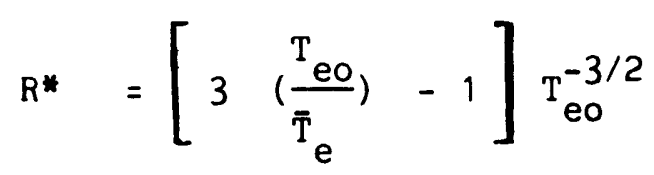

The function $x$ is proportional to the cross sectional area, which depends upon the elongation and triangularity of the plasma. It is given by the exp.ession

$$
x=\equiv \kappa\left(R_{0} / A\right)^{2}\left[J_{0}(d)+J_{2}(d)\right],
$$

where $k$ is the elongation, $J_{0}$ and $J_{2}$ are the spherical Bessel functions, and $d$ is the triangularity [2]. We now wish to replace Wesson's function, $g(Z)=$ $N(Z) Z$, with some convenient expression depending upon $Z_{\text {eff }}$ A reasonable fit for $g(Z)$ versus $Z$ yields

$$
g(2)=.591(1+2) \text {. }
$$

The expression for the resistance is thus

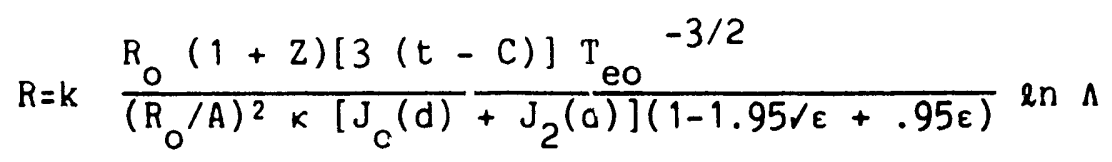

where $t=\mathrm{T}_{e o} / \overline{\mathrm{T}}_{\mathrm{e}}$, and $\mathrm{C}=1 / 3$. 
A detailed calibration of this equation against results taken from RIP simulations of various DIII-D, ITER, and ARIES plasmas revealed that a better fit is found with

$$
\begin{aligned}
& k=1.255 \times 10^{-9} \\
& C=0.623+0.012 \mathrm{~T}_{\text {eo }}
\end{aligned}
$$

Notice that $C$ deviates from the previous value of $1 / 3$ to approximately 1.0 for $\mathrm{T}_{\text {eo }} \approx 30 \mathrm{keV}$. It is found best to evaluate $Z$ for multiple ions as $Z \equiv Z$ eff $=\Sigma n_{j} \mathrm{j}^{2 / n_{e}} \cdot$

Table 2 is a detailed summary of ohmic resistance calculations taken from RIP simulations and the corresponding resistances calculated from equation (21). The results from the model are then plotted against those from RIP in Figure 3. Most values agree within $10 \%$ of each other with the one exception of the first ARIES run where the model differs from RIP by more than $50 \%$. However, this is understandable since the safety factor was not monotonic for the equilibrium solution. This then serves as a necessary requirement for the validity of this model.

\subsection{Resistance In An RF Plasma}

Since our primary interest is in those plasmas heated and/or driven by $\mathrm{RF}$ waves, it is worth discussing the validity of our expression for the resistance when we allow for coupling of $\mathrm{RF}$ and inductive effect such that the total current is given by $\mathrm{J}_{\mathrm{TOT}}=\mathrm{J}_{\mathrm{OH}}+\mathrm{J}_{\mathrm{KF}}+\sigma_{1} \mathrm{E}+\ldots$ where we have included the additional current, $\sigma_{1} E$, described by Fisch. This term arises when both RF power and inductive effects are present. Using Fisch's expression for the hot conductivity 


$$
\begin{aligned}
& \sigma_{1}=\sigma_{\mathrm{Sp}}\left(1+\frac{2}{3+\mathrm{Z}}\right) \frac{2+.72}{32 \sqrt{2 \pi}} \frac{\mathrm{J}_{\mathrm{RF}}}{\mathrm{env}_{\mathrm{T}}} \mathrm{W}^{2}, \\
& \text { it can be shown that for a typical DIII-D plasma where } \mathrm{J}_{\mathrm{RF}}=5 \times 10^{5} \mathrm{~A} / \mathrm{m}^{2} \text { and } \\
& W=v_{\|} / \mathrm{v}_{\mathrm{T}}=3 \\
& \sigma \simeq 10^{-2} \sigma_{\mathrm{sp}}
\end{aligned}
$$

Neglecting $\sigma_{1} E$ then allows us to determine $I_{O H}=V_{L} / R$ from Ohm's law

$$
I_{\text {TOT }}=\frac{V_{L}}{R}+I_{R F}
$$

assuming that $v_{\|}<v_{R}$. For DIII-D, the plasma current is on the order of several hundred kiloamperes and the electric field is small enough that $v_{R}$, the runaway speed, is near the speed of $l$ ight and $v_{\|} \approx \frac{1}{4} v_{R}=7.5 \times 10^{7} \mathrm{~m} / \mathrm{s}$.

Our model for the ohmic resistance proves to be a reasonably accurate and convenient tool for studying tokamak burn scenarios where the electric field is small and $v_{\|}<v_{R}$.

\section{REFERENCES}

1. D.A. Ehst, Nucl Fusion $\underline{25}$ (1985) 629.

2. D.A. Ehst, K. Evans, Jr., Nucl. Fusion 27 (1987) 1267.

3. N.J. Fisch, Phys. Fluids 28 (1985) 245.

4. D.A. Enst, C.F.F. Karney, Nucl. Fusion, to be published.

5. S.P. Hirshman and D.J. Sigmar, Nucl. Fusion 21 (1981) 1079.

6. F.L. Hinton and R.D. Hazeltine, Rev. Mod. Phys. 48 (1976) 239.

7. J. Wesson, "Tokamaks," Oxford Press (1987). 

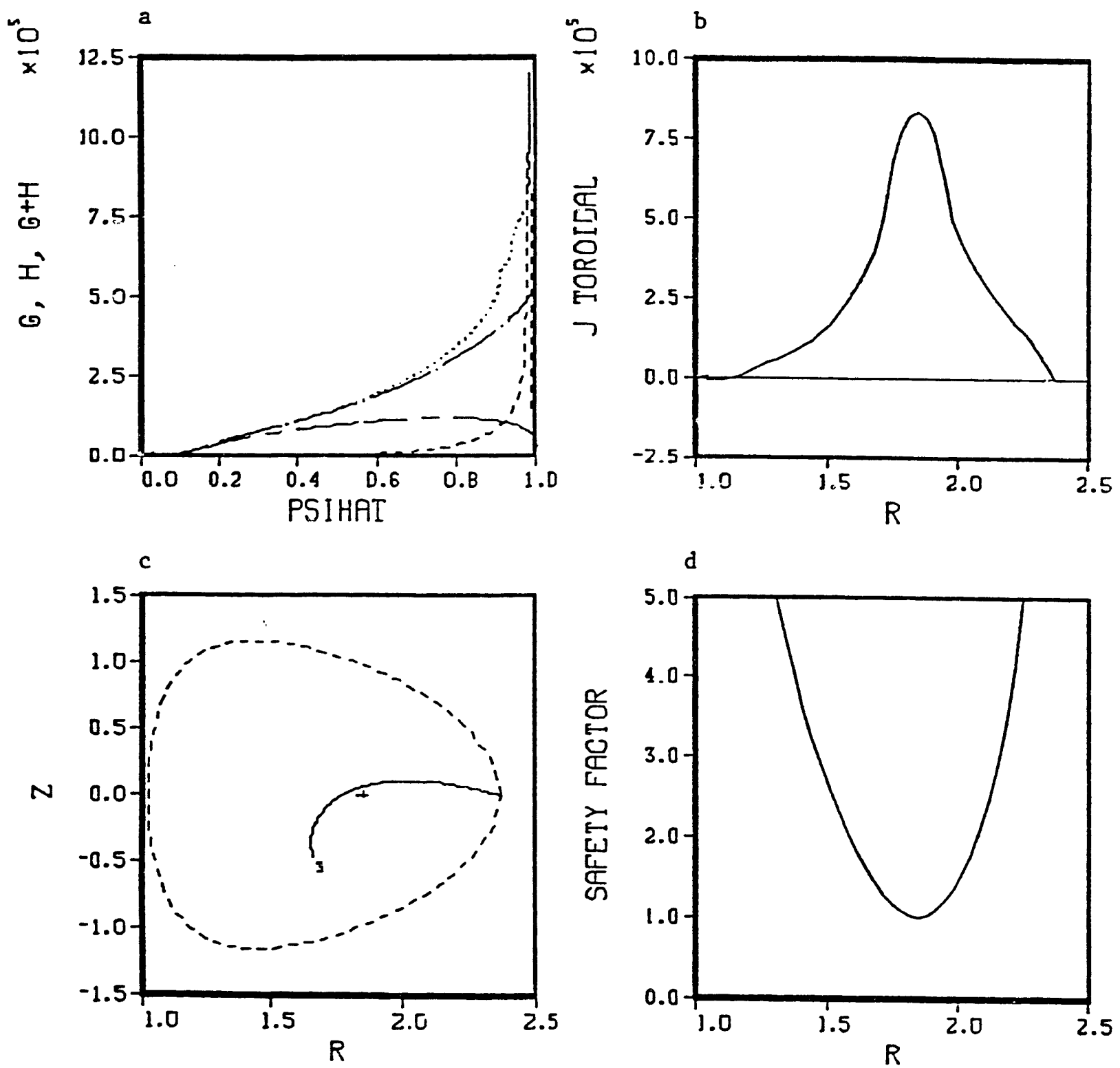

Figure 1. RFCD/MHD equilibrium for DIII-D with $T_{e o}=3.5 \mathrm{keV}, \mathrm{n}_{e 0}=0.2 \mathrm{x}$ $10^{20} \mathrm{~m}^{-3}$. (a) Contributions to $\langle J, B\rangle$ from FWCD (dashed), bootstrap (chain-dashed), inductive + bootstrap (chain-dotted), and total (dotted). (b) Components of toroidal current density. (c) FW ray trajectory. (d) Safety factor. 


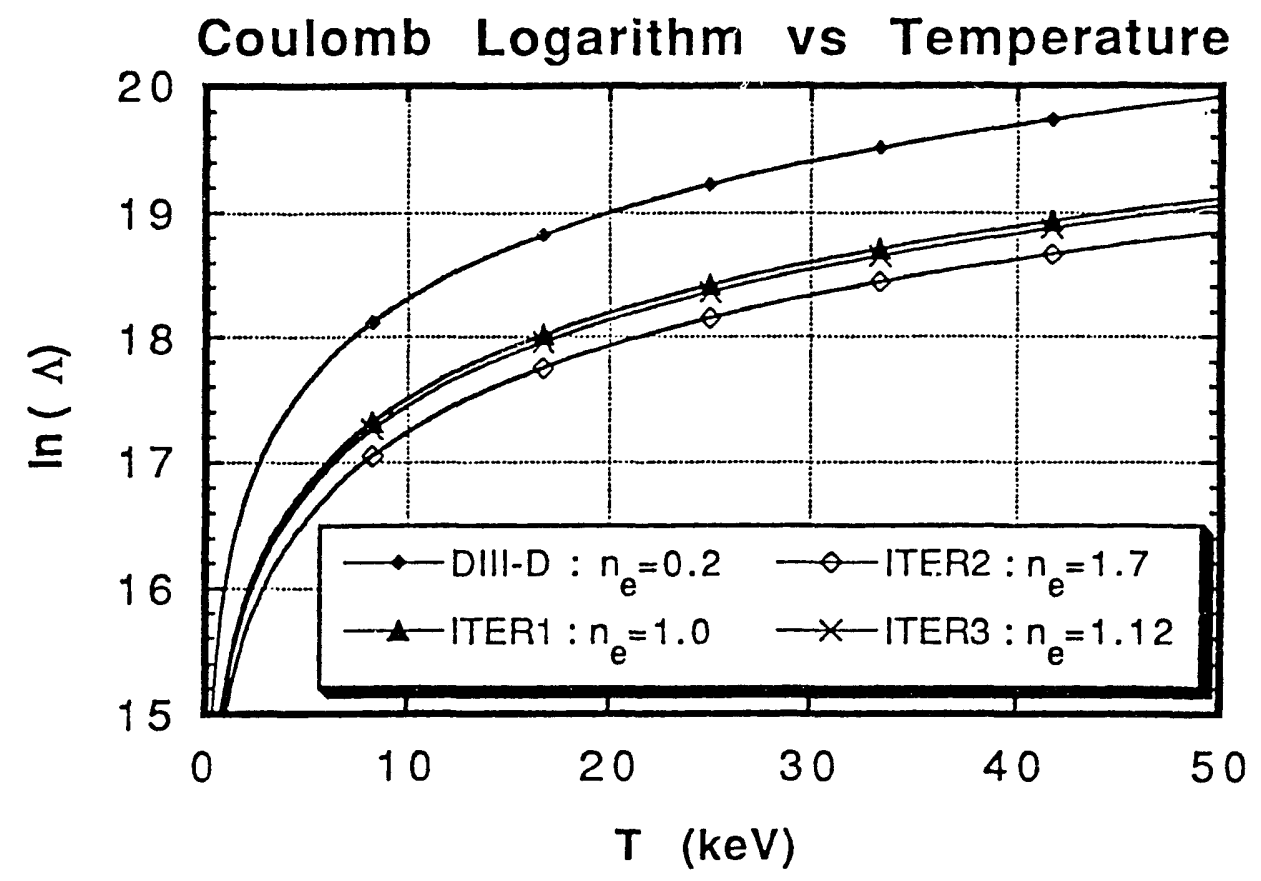

Figure 2. Coulomb Logarithm.

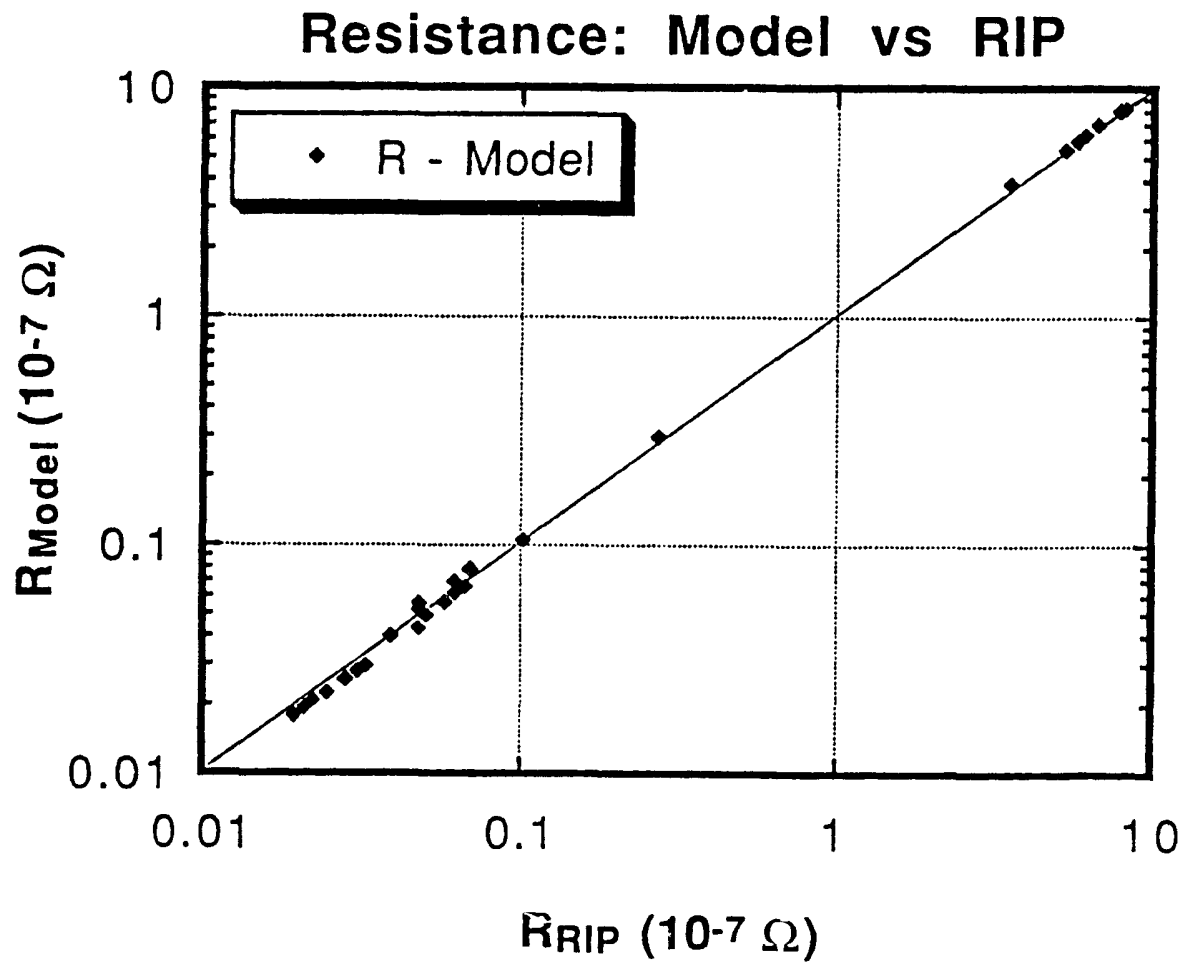

Figure 3. Comparison of analytic and numerical resistance. 


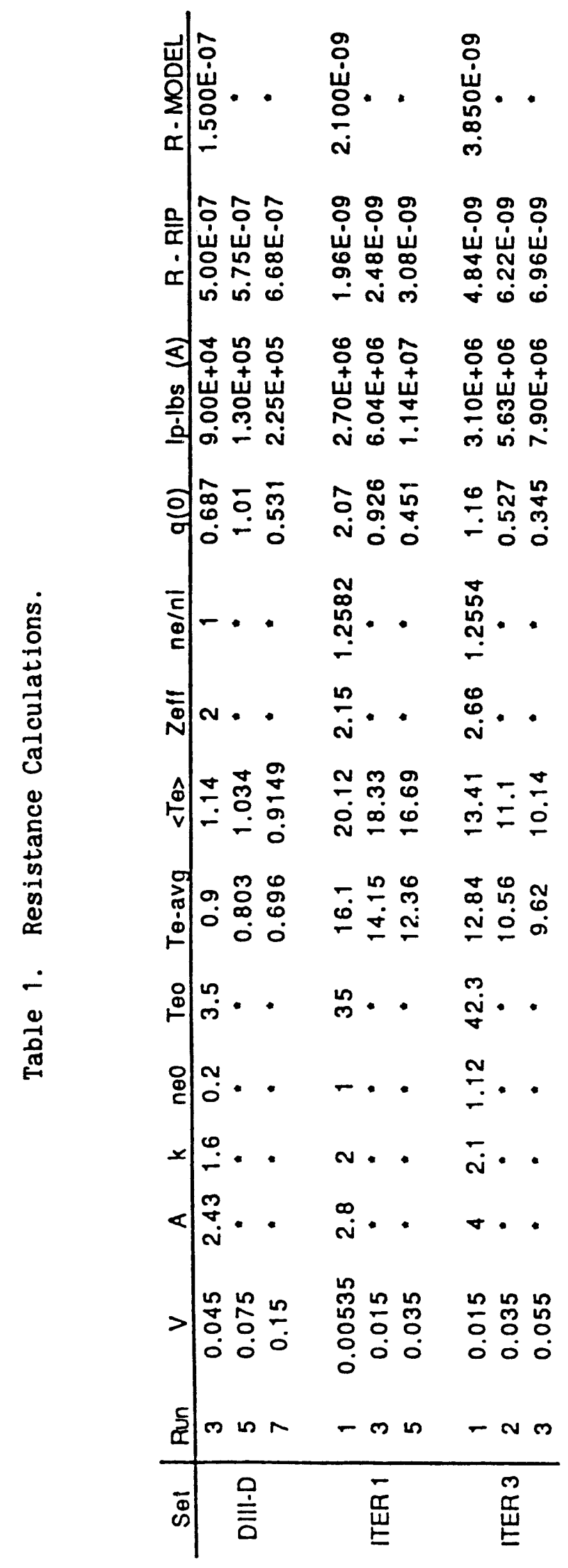




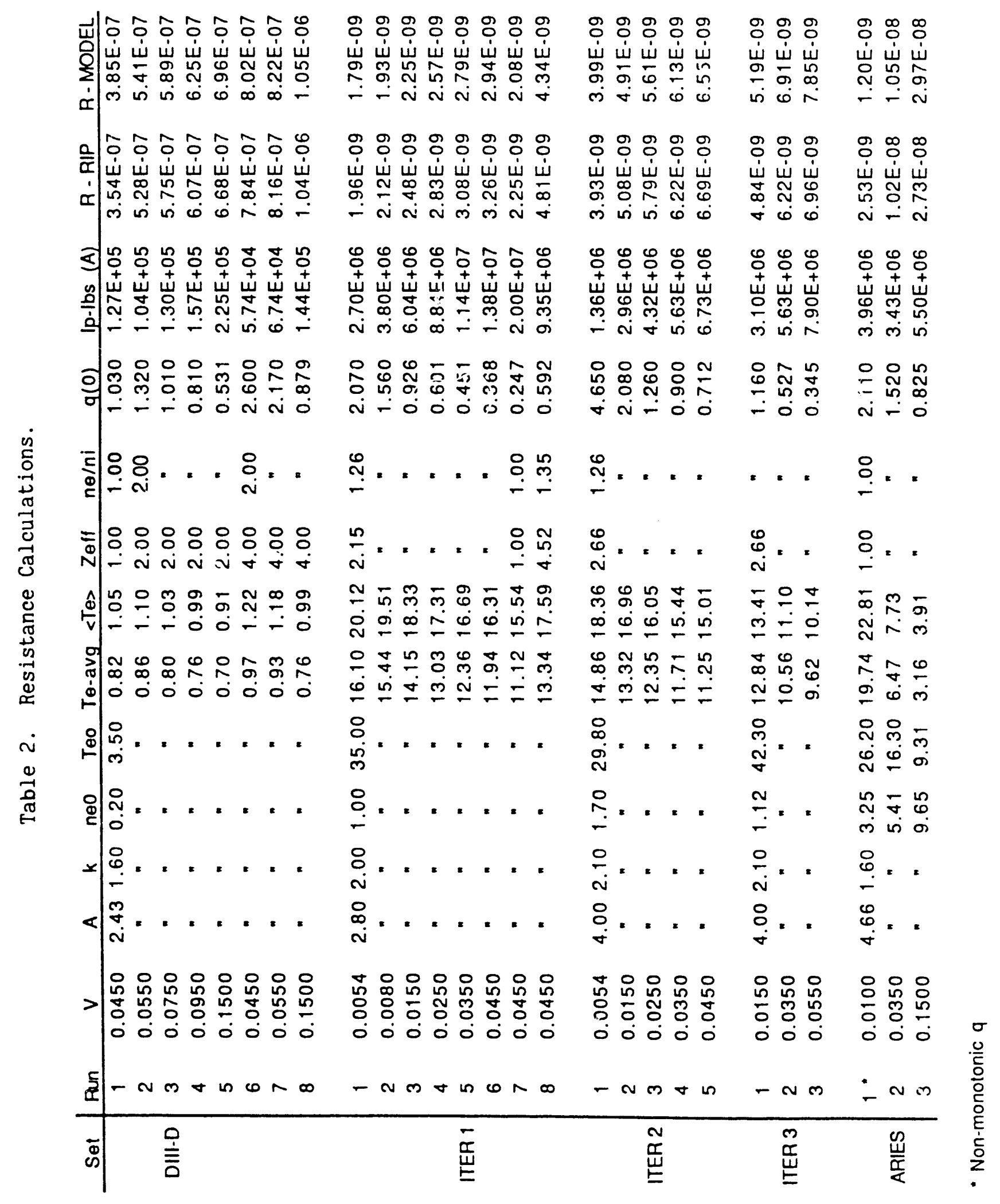




\section{DISTRIBUTION LIST OF ANL/FPP/TM-256}

\section{Internal}

$\begin{array}{ll}\text { H. Attaya } & \text { T. Hua } \\ \text { J. Brooks } & \text { R. Mattas } \\ \text { H. Chiu } & \text { B. Picologlou } \\ \text { D. Ehst } & \text { C. Reed } \\ \text { K. Evans } & \text { D. Smith } \\ \text { P. Finn } & \text { D. -K. Sze } \\ \text { Y. Gohar } & \text { L. Turner } \\ \text { A. Hassanein } & \end{array}$

FPP Files (10)

ANL Contract File

TIS Files (3)

ANL Patent Dept.

\section{External}

DOE/OSTI for distribution per UC-420 (40)

Manager, Chicago Operations Office-DOE

ANL-TIS Libraries

C. Bathke, Los Alamos National Laboratory

S. Berk, Of fice of Fusion Energy/U.S. Department of Energy

C. Bolton, Orfice of Fusion Energy/U.S. Department of Energy

L. Bromberg, Massachusetts Institute of Technology

D. Cohn, Massachusetts Institute of Technology

R. Conn, University of California, Los Angeles

D. Crandall, Office of Fusion Energy/U.S. Department of Energy

R. Dagazian, Office of Fusion Energy/U.S. Department of Energy

J. Hogan, Oak Ridge National Laboratory

S. Jardin, Princeton Plasma Physics Laboratory

J. Kinsey, University of Illinois, Urbana

R. Krakowski, Los Alamos National Laboratory

D.M. Meade, Princeton Plasma Physics Laboratory

W.M. Nevins, Lawrence Livermore National Laboratory

E. Cktay, Office of Fusion Energy/U.S. Department of Energy

J. Perkins, Lawrence Livermore National Laboratory

M. Peng, Fusion Engineering Design Center

M. Phillips, Grumman Corporate Research Center

D. Post, Princeton Plasma Physics Laboratory

$\mathrm{J}$. Ramos, Massachusetts Institute of Technology

P.H. Rutherford, Princeton Plasma Fhysics Laboratory

J. Schultz, Massachusetts Institute of Technology

C. Singer, University of Illinois, Urbana

W. Stacey, Georgia Institute of Technology

D. Steiner, Rensselaer Polytechnic Institute 

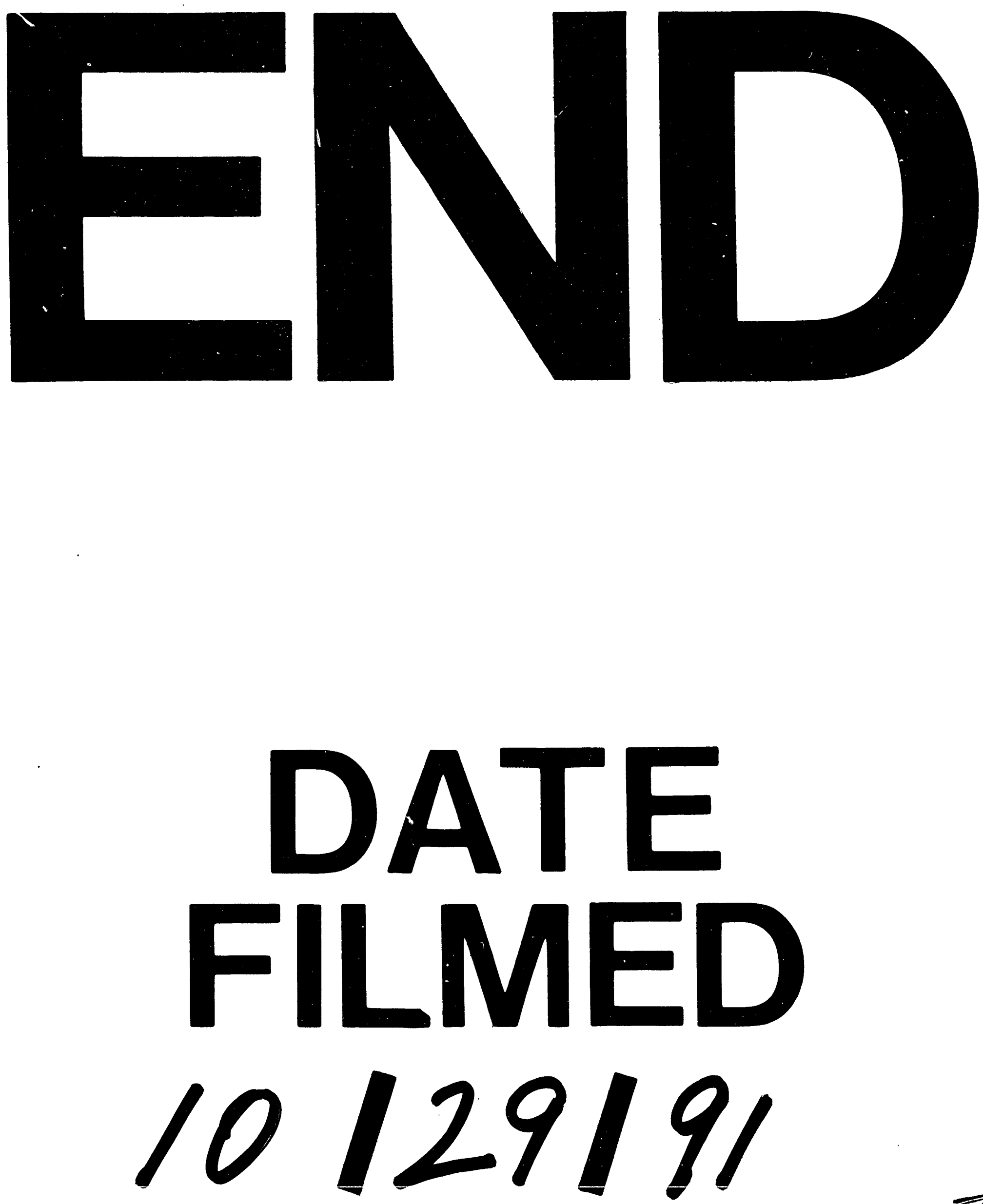
